\title{
WARGAMING AND CHALLENGES IN THE EXPERIMENTATION DOMAIN
}

\section{Jan HODICKY}

\author{
Centre for the Security and Military Strategic Studies at the University of Defence, \\ Brno, The Czech Republic \\ jan.hodicky@seznam.cz
}

\begin{abstract}
Military domain lacks the sufficient funding. It becomes extremely critical investing into the right projects that will deliver the expected outcome in the acquisition phase from the medium and long term perspective. The Concept Development and Experimentation (CD\&E) is a powerful approach to reduce the related risk if it is consistently applied. Wargaming is a method that is used in the military to get insight about the examined phenomena when employing the military decision making process. The article deals with the role of wargaming in the CD\&E. The second part of the article describes the architecture of wargame, main constructs, phases and critical factors of its design. The last part of the article describes the proposed Case Study of the Computer Assisted Wargaming $(C A W)$ to be used for the complex problem like military capability planning and risk reduction under the specified uncertainty. Uncertainty is reduced by proper Design of Experiment when the CAW plays the crucial role.
\end{abstract}

Keywords: modelling and simulation, wargaming, experimentation, capability planning

\section{Introduction}

Military budget is very limited in the most European countries and goes below the 2\% of the Ground Domestic Product [1]. The only approach in the current political and economic situation is to implement into $\mathrm{MoD}$ strategic decision making process tools/approaches that might help to reduce the cost and mainly uncertainty related to the wrong implementation of such decision into the defence planning. It becomes extremely critical investing into the right projects that will deliver the expected outcome in the acquisition phase from the medium and long term perspective. The Concept Development and Experimentation (CD\&E) approach is at the moment the most effective and accepted means to support the capability planning as the main objective of defence planning. CD\&E defines 14 principles to follow and five main components of any Experiment [2]. The components are described as:

- Independent variable or treatment. Input variables that may influence warfighting effectiveness.

- Dependent variable or effect. Output variables that are results of the trial and shows potential increase or decrease in some measure of warfighting effectiveness.

- Experimental unit or team that executes the trial.

- Trial is one observation of the experimental unit under one alternative of independent variables to see the effects on the dependent variables.

- Analysis compares the results of all trials in the experiment.

CD\&E should employ one of the following methods: 
- Live Experiment. A trial is done in the live environment, real soldiers and real capability is involved; only the effects might be simulated.

- Manual Wargame. A trial is executed in the form of wargame. The adjudication about the results of the Course of Action (CoA) is done manually by arbiter/ umpire.

Computer Assisted Wargame (CAW). wargame. The adjudication about the results of the CoAs and the execution of wargaming is supported by computers; it means simulation might be used as one of the operation research approaches [4]. A method of the human in the loop in is employed. Therefore the results of the trial are translated by the human being and human factors are included.

A trial is performed in the form of a

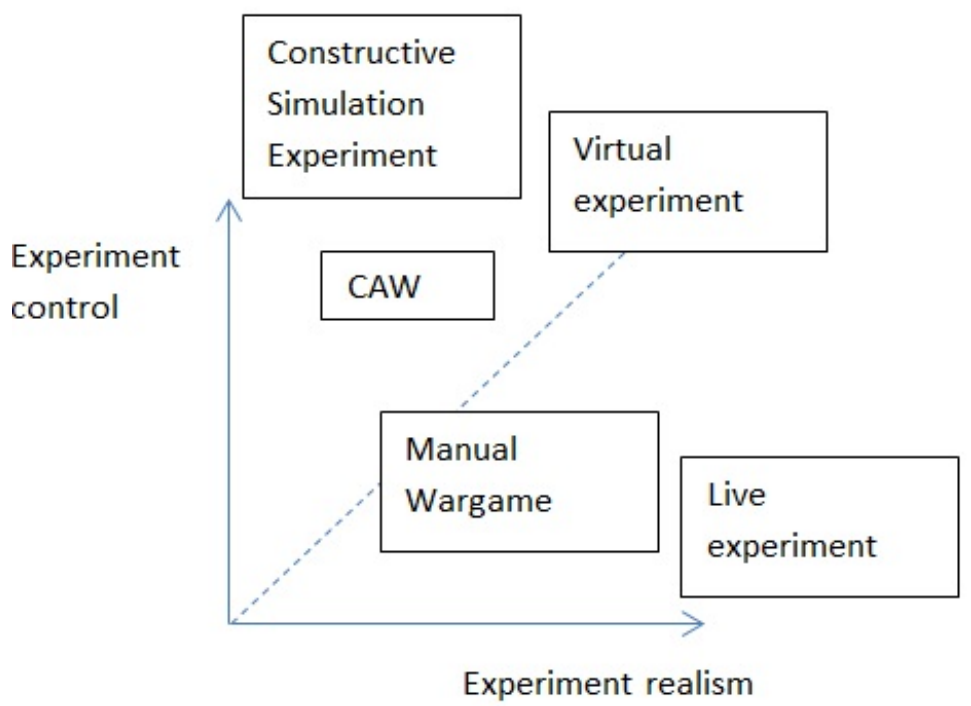

Figure 1: Experimentation and scope of methods

- Constructive Simulation Experiment. A Trial is done in synthetic environment created by constructive simulation [3]. Capabilities and human decision is fully replicated in the constructive simulation. Human input is limited to creation of CoAs of players. Important feature is limited number of operators to be able to manage a simulator effectively.

- Virtual Experiment. A trial is carried out in the virtual environment, where actors are mixture of real person in the avatar form and artificial intelligence. Decision about CoAs might be done by simulator or by human- avatars.

Figure 1 describes the scope of the method and its relation to the experiment control and experiment realism. The best way to control the experiment and assure the rigor from the procedural point of view is to use a constructive simulation. This means eliminates any non-wanted human decision and allows executing the experiment in very structured way. However the realism is a critical element. Human decision is limited to the capacity of artificial intelligence implemented in the simulation because there is no human being interaction during the execution of a trial.

On the other side there is the Live Experiment where the realism is very high. However the way to control the experimental trial is limited. Another important factor is that live experiments are usually very expensive and an element of a danger shouldn't be underestimated either. CAW and manual war gaming seem to be the optimum solution to support analytical studies in defence from the applicability and resources point of view.

Another solution that brings good ration 
between the experiment control and it realism is to perform a trial as complete Virtual Experiment; however the current state of the art in the simulation domain is not mature enough. It would require connecting the virtual environment where all players are in the form of avatars with the adjudication platform instantiated by simulation.

\section{Wargaming as an analytical tool}

The history of wargaming is connected with Kriegsspiel. It was the very first systematic approach to train a commander staff in decision making process in the Prussian army in 1830 . It was originally played as a game strictly controlled by explicitly defined rules. However it proved to be contra productive. The players abused the rules to win the game and there was no authority - umpire who decides on the results of the particular CoA. Therefore the open version of the Kriegsspiel was launched and rules were more flexible and adjudication of CoA was done by a senior rank with sufficient authority. From definition of wargaming as "A warfare simulation that does not involve the actual forces, using rules data and procedures in which the flow of events shapes and is shaped by decisions made by a human player or players", is evident that individual decision is made by human being or other players. The meaning of the others is the artificial intelligence. Word simulation explains that decision making process is done over the simulated environment, even if an experiment might be run as live one.

Another important fact is that a flow of incidents is controlled by participants, it is not pre-scripted. However, good scripting is needed in the design phase of wargaming to set up the scene.

Wargaming is a process composed of four main steps [5]:

- Set up of preconditions. The experimental unit must be trained in using WG and personnel running $\mathrm{WG}$ as well.

- Preparation and planning. Documentation like scenarios, responsibilities, Main Event List (MEL)/ Main Incident List (MIL) must be prepared well in advance.

- Execution. Execution of WG is usually run in sequences that correspond to the identified scenarios/ vignettes. Each sequence is run in one or more cycles depending on the level of expected insight/analysis. Each cycle is run as the process described on the Figure 2. A cycle is composed of five steps. Initial situation is explained by facilitator and stress is put on the situation descried by a vignette. It is followed by $\mathrm{CoA}$ proposed by Blue Team with detailed description over the map to other participants. Then Red Team explains its CoA and Bluet Team react in a new counter CoA. The very last part of the cycle is the cognition phase run by umpire and facilitator where the main insight about the cycle is amplified.

- Evaluation of collected information. After action review and analytical part must be done at the end of any WG activities. A quality of this phase influences an acceptation of the results of WG process, mainly if it used for analytical purposes.

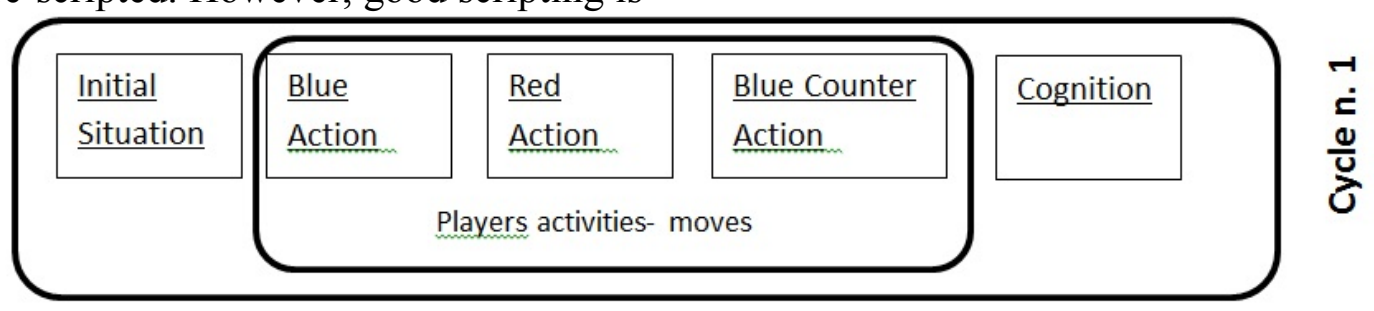

Figure 2: WG execution phase 
Wargaming is relatively cheap, and open to innovative way of thinking, method that allows educating, training, informing, and analyzing of a problem domain. In further reading the stress is put on the analytical capability of wargaming where the objective is to make recommendation for decision making process based on the results of the wargaming process.

\section{CAW architecture}

CAW architecture is composed of the following constructs:

- Scenario. The main driver of wargaming process is an exhaustive description of the scene via a scenario. The scenario must be transferred from the language of military to the executable form readable by computer; e.g. Military Scenario Description Language [6].

- ORBAT. Order of Battle contains the current capability of own forces and its characteristics.

- Objectives. WG design must start with explicit definition of its objectives.

- Maps and Charts. WG must be run over the map or replication of the map by current Information and Communication Tools like, Geographical Information System (GIS) or 2D/3D modelers.

- Clock. Wargaming is not an activity running in continues manners. It is executed in discrete time intervals. Therefore a clock is represented by Simulation time that is changed in the pace required by the Players to better understand a problem domain.

- Rules\&Data. The adjudication over players' moves is done by umpire that follows one part rules that are explicitly stated and the second one that is based on the umpires $\backslash$ best knowledge. The first category of rules might be coded in the core of a simulation system to support a decision of an umpire.

- Players. Dynamicity of a wargame is created by players. They might be in the form of a human being or an artificial intelligence, representing both or one side.

- Analysts. The role of analyst is crucial mainly in the situation when the WG is used for analytical purposes.

- Facilitator. The role of facilitator is to support flawless execution of the game by giving assistance to the players to better achieve the objectives of the WG.

- Umpire. The role of the umpire is to make decision over the executed CoA and to support cognition phase of each cycle of the execution phase.

\section{Critical factors in WG}

Application of WG method generally depends on many factors; the most important and challenging are elaborated bellow:

- Experienced umpire/ facilitator. The umpire must be a senior officer who is accepted as the character in the military domain by the players and is experienced in WG execution. Facilitator must be trained in WG execution, however more critical for him is to understand the objective and scenario of WG in the very detailed way.

- Collaborating staff. Players must be motived to be part of the process and should be nominated from the very beginning of design of $\mathrm{WG}$ and not changed in the middle of the WG process.

- Scenario. Scenarios must be developed as close as possible to the reality; otherwise it is going to be refused by the players. If it is run as CAW then the scenario must be replicable in the synthetic environment, e.g. in the constructive simulation.

- Feedback and Records (FIR, AAR). For analytical purposes and for justification of WG design and execution expenditures, immersion of feedbacks' collection must be done in very sensitive and rigorous way. Otherwise it might spoil a flow of the execution and it might 
create barrier in the freedom of players' innovative thinking.

- Visualization. The game is played over terrain that needn't to be only in the form of a cart map but more often is visualized in $2 \mathrm{D}$ or $3 \mathrm{D}$. It brings a new challenge in the WG design and execution. Critical factor is to find out limits on the level of details to be displayed to the players.

- Working Information and Communication Technology (ICT). CAW is founded on the support from ICT world. Therefore up and running, stable and flexible ICT environment must be implemented; otherwise it might be even contra productive.

- Precise planning phase of WG. Quality of the execution phase of WH is highly influenced by the planning phase. All documents must be prepared in high quality a consistent manner. Details are important, even if the $\mathrm{WG}$ is run at the strategic level.

- Entertainment. The last but the most important factor in WG execution is amusement. If players are not attracted by scenarios, if they do not feel to be part of the group, if the map model in 2D or $3 \mathrm{D}$ is not eyes' catching, then the objective of the WG is difficult to achieve. Facilitator plays here important role as well.

\section{Proposed Case Study}

The Czech Armed Forces doesn't have at the moment formalized procedure to deal with capability planning. The objective of the current research is to use the power of analytical tools like CAW and to implement it into a new capability planning process in accordance with CD\&E approach. Figure 3 describes proposed architecture of applied CAW in the capability planning.

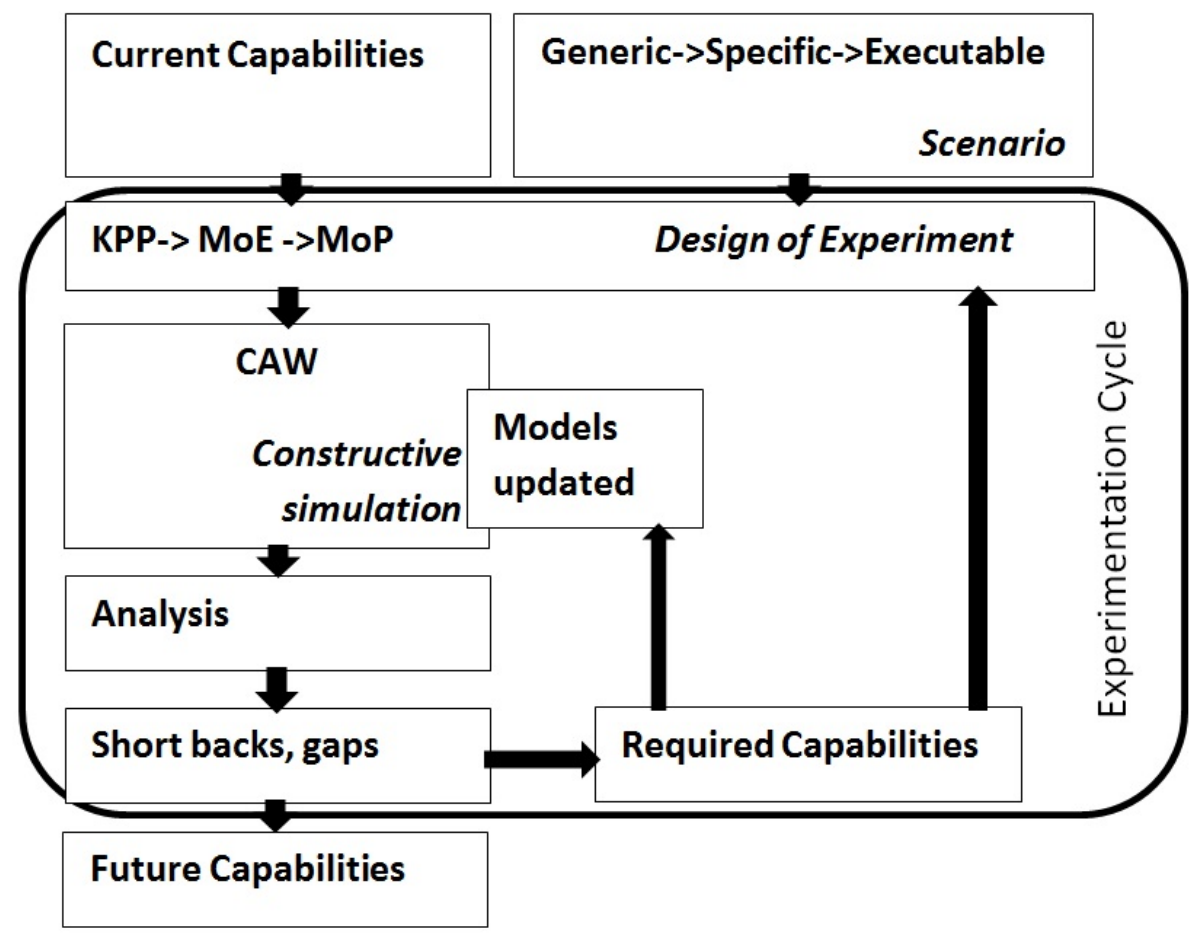

Figure 3: WG execution phase

Main input in the architecture is scenario at the strategic level. It must be translated into the executable form [6], [7] readable by constructive simulation used as a core of analytical method of CAW [8], [9], [10]. Second main input is the current capability that is available in the moment of the capability planning process. It creates condition for Design of the Experiment (DoE) where Key Performance Parameters (KPP) at the strategic level through Measure of Effectiveness (MoE) at the 
operational level up to the Measure of Performance (MoP) at the system/model level are defined. Next phase is the first cycle of wargaming with objective to find out gaps and short back in the Current Capabilities in confrontation to defined Scenarios. Analysis enables transferring the results of the CAW from the System Level represented by MoP back up to the strategic level and KPP. If gaps identified a new experimentation cycle must be run and models must be updated in the constructive simulation to adequately represent new capabilities required. Then DoE might be updated based on new set of MoPs. The Experimentation cycles are repeated till the moment the Future Capabilities are clearly identified.

\section{Conclusions}

The role of experimentation is crucial in any complex military environment where decision is needed and parameters are not clear, the problem domain is not well defined and factor of uncertainty is very high. Implemented process of capability planning supported by CAW will increase confidence that the findings are valid and creates a systematic body of knowledge to inform and investigate capability development. The future work will be focused on the development of the strategy to translate scenarios from generic description down to the executable form and on definition of the set of Key Performance Parameters, Measure of Performance and Measure of Effectiveness to unify the inputs for the capability planning process.

\section{Acknowledgements}

This work is sponsored by the project called STRATAL (2016-2020).

\section{References}

[1] Mironova, D.S. Nato transformation in the context of global financial and economic crisis (2016) World Economy and International Relations, 60 (6), pp. 80-89.

[2] Guide for Understanding and Implementing Defense Experimentation (GUIDEx). ISBN 92-95046-11-0. Canadian Forces Experimentation Centre, Ottawa, Canada. 2006.

[3] Hernandez, A.S. Integrating simulation-driven decisions and business wargames to shape fiscal policies (2015) Simulation Series, 47 (2), pp. 111-118.

[4] Novotny, Antonin; Prochazka, Dalibor. Application of system dynamics in the process of sharing military capabilities. In: Proceedings International Scientific Conference STRATEGIES XXI The complex and dynamic nature of the security environment. Bucharest, Romania: Carol I National Defence University Centre for Defence and Security Strategies Studies, 2015, p. 90-101. ISSN 2285-8318.

[5] Dunnigan, J.F. (1992) The Complete Wargames Handbook.

[6] Blais, C., Dodds, R., Pearman, J., Baez, F. Rapid Scenario Generation for multiple simulations: An application of the Military Scenario Definition Language (MSDL) (2009) Simulation Interoperability Standards Organization - Spring Simulation Interoperability Workshop 2009, pp. 20-32.

[7] McCreight, R. Scenario development: Using geopolitical wargames and strategic simulations (2013) Environmentalist, 33 (1), pp. 21-32.

[8] Casar, J., Stefek, A.: Analysis of decision process in HLA simulations. Croatian Journal of Education, 2012, vol. 14, no. Special Edition 1/2012, p. 21-24. ISSN 1848-5197.

[9] Casar, Josef. Expert analysis of decision process in simulator In: international conference $6^{\text {th }}$ Advance in Mechatronics 2011. Brno: Expertia o.p.s., 2011, p. 0-0. ISBN 978-80-7231-848-3.

[10] STODOLA, Petr; MAZAL, Jan. Tactical Decision Support System to Aid Commanders in their Decision-Making. In: Modelling and Simulation for Autonomous Systems. Rome: Springer, 2016, p. 396-406. ISSN 0302-9743. ISBN 978-3-319-47604-9. 